\section{Are These Truly Rheumatoid Arthritis or Antisynthetase Syndrome Cases?}

\author{
Sertaç $\operatorname{KETENCI}^{1}$ (D), Ender SALBAŞ² (D) \\ ${ }^{1}$ Giresun University Medical Faculty, Rheumatology, Giresun, \\ Turkey \\ ${ }^{2}$ Ömer Halisdemir University Bor Physical Therapy and \\ Rehabilitation Education Research Hospital, Physical Therapy \\ and Rehabilitation, Niğde, Turkey
}

\section{Dear Editor,}

We read with interest the study of Matsushida and colleagues entitled "The Association of AntiAminoacyl-Transfer Ribonucleic Acid Synthetase Antibodies in Patients With Rheumatoid Arthritis and Interstitial Lung Disease" ${ }^{1}$ published in your journal.

We would like to inform you of some of the issues that drew our attention in the related work.

In the study, 228 patients classified as rheumatoid arthritis (RA) according to the 2010 American College of Rheumatology (ACR)/ European League Against Rheumatism (EULAR) classification criteria were included. The aim of the 2010 criteria is to provide early recognition of RA cases and initiation of treatment before structural damage. For this reason, the specificity of the criteria is criticized and the possibility of other rheumatologic pathologies in the early period being misconsidered as RA is emphasized. ${ }^{2}$ As a matter of fact, in a study of Brittsemmer et al. ${ }^{3}$ with 455 early arthritis patients, although $51 \%$ patients were classified as RA with current criteria, they were defined as non-RA according to expert opinion. For this reason, we believe that the anti-aminoacyl-transfer ribonucleic acid synthetase (ARS) positive patients described by the authors in the study should be examined for whether or not they really had RA.

Antisynthetase syndrome (AS) is an important clinical condition in the differential diagnosis of early RA. It has been reported that development of myositis can take years after onset of arthritis and interstitial lung disease (ILD), which are also not rarely seen before myositis. ${ }^{4,5}$ Of the cases, 10\% have disease with other findings and myositis never occurs. ${ }^{6}$ The expected pattern of arthritis in AS is polyarthritis in which small joints such as wrist metacarpophalangeal and proximal interphalangeal are symmetrical which is quite similar to RA joint involvement. ${ }^{7}$ Anti-cyclic citrullinated peptide (CCP) and rheumatoid factor (RF) positivity can be observed in AS and lead to false RA diagnoses. ${ }^{8}$ On the other hand, we do not have established knowledge that ARS is seen in cases with classical RA.

In the study of Matsushida et al., ${ }^{1}$ non-specific interstitial pneumonitis (NSIP) was reported as ILD type all over the ARS positive patients classified as RA. NSIP is the most common pattern of pulmonary involvement in patients with AS. ${ }^{9}$ However, the most common pattern of pulmonary involvement that is expected to be seen in RA cases is usual interstitial pneumonia and it is not found as the most common form of pulmonary involvement in the relevant article of Matsushida et al. ${ }^{1}$

The authors noted that biological agents in the treatment of myositis exacerbated the disease and that there was no increase in disease activity in their present cases. Biological treatments used by the patients are as follows: abatacept in two

Received: April 05, 2018 Accepted: April 23, 2018 Published online: August 16, 2018

Correspondence: Sertaç Ketenci, MD. Giresun Üniversitesi Tıp Fakültesi Romatoloji Bilim Dalı, 28200 Giresun, Turkey Tel: +90 507 - 8665533 e-mail: drsertacketenci@hotmail.com 
cases and etanercept in one case. Abatacept is a successfully tested agent in resistant myositis. ${ }^{10}$ Biological treatments accused of exacerbation in myositis are usually anti-tumor necrosis factor agents. ${ }^{11}$ It has been reported in the current study that; in one case treated with etanercept, exacerbation in disease clinic did not seen. However, we do not consider "no increase in disease activity" as evidence for the diagnosis of RA.

For all these reasons, we believe that more serious evidence is needed to show that all cases in this article were true RA. We think that the authors should show how long they followed-up these cases, how many patients did not develop myositis, how many patients developed myositis, whether there were typical erosive findings seen in RA, and whether there were Raynaud's phenomenon and capilleroscopic changes in these patients that are more common in myositis.

\section{Declaration of conflicting interests}

The authors declared no conflicts of interest with respect to the authorship and/or publication of this article.

\section{Funding}

The authors received no financial support for the research and/or authorship of this article.

\section{REFERENCES}

1. Matsushita M, Tamura N, Ogasawara M, Tada K, Yamaji K, Takasaki Y. The Association of AntiAminoacyl-Transfer Ribonucleic Acid Synthetase Antibodies in Patients With Rheumatoid Arthritis and Interstitial Lung Disease. Arch Rheumatol 2018;33:26-32.

2. Kennish L, Labitigan M, Budoff S, Filopoulos MT, McCracken WA, Swearingen CJ, et al. Utility of the new rheumatoid arthritis 2010 ACR/EULAR classification criteria in routine clinical care. BMJ Open 2012;2(5). pii: e001117.

3. Britsemmer K, Ursum J, Gerritsen M, van Tuyl LH, van Schaardenburg D. Validation of the 2010 ACR/ EULAR classification criteria for rheumatoid arthritis: slight improvement over the 1987 ACR criteria. Ann Rheum Dis 2011;70:1468-70.

4. Katzap E, Barilla-LaBarca ML, Marder G. Antisynthetase syndrome. Curr Rheumatol Rep 2011;13:175-81.

5. Patel HC, Lauder NN. The antisynthetase syndrome. Am J Med 2011;124:e3-4.

6. Tzioufas AG. Antisynthetase syndrome. Orphanet Encyclopedia 2001:1-5.
7. Solomon J, Swigris JJ, Brown KK. Myositis-related interstitial lung disease and antisynthetase syndrome. J Bras Pneumol 2011;37:100-9.

8. Mumm GE, McKown KM, Bell CL. Antisynthetase syndrome presenting as rheumatoid-like polyarthritis. $\mathrm{J}$ Clin Rheumatol 2010;16:307-12.

9. Zamora AC, Hoskote SS, Abascal-Bolado B, White $\mathrm{D}$, Cox CW, Ryu JH, et al. Clinical features and outcomes of interstitial lung disease in anti-Jo-1 positive antisynthetase syndrome. Respir Med 2016;118:39-45.

10. Tjärnlund A, Tang Q, Wick C, Dastmalchi M, Mann $\mathrm{H}$, Tomasová Studynková J, et al. Abatacept in the treatment of adult dermatomyositis and polymyositis: a randomised, phase IIb treatment delayed-start trial. Ann Rheum Dis 2018;77:55-62.

11. Dastmalchi M, Grundtman C, Alexanderson H, Mavragani CP, Einarsdottir H, Helmers SB, et al. A high incidence of disease flares in an open pilot study of infliximab in patients with refractory inflammatory myopathies. Ann Rheum Dis 2008;67:1670-7.

\section{Author Response}

Thank you very much for your valuable feedback.

You raised some concerns about whether the cases reported in our study were authentically RA, or actually antisynthetase syndrome characterized by symptoms of arthritis or ILD. We would like to express our appreciation for your informative remarks. We too understand the importance of this point: in fact, this similarity complicated our efforts to diagnose some of the cases in the study. Nevertheless, we included these cases in our report because we deemed a diagnosis of RA to be reasonable at the time of our investigation.

Our impetus for starting this research was a RA patient who exhibited persistent polyarthralgia, ulnar deviations, and swan neck deformities in the fingers of both hands, as well as X-ray findings of bone erosion and destruction, who additionally tested positive for anti-PL-12, a kind of anti-aminoacyl-transfer ribonucleic acid synthetase (ARS) antibody. We diagnosed her with classic RA based on hematological findings of anti-CCP and RF positivity, and high levels of metalloproteinase-3 C-reactive protein, and other markers. This patient also had comorbid ILD, which led us to carefully examine her condition using bronchoscopy; yet, we observed no signs of concurrent polymyositis or dermatomyositis. 
Based on our experience with this patient, we measured anti-ARS levels in many patients with classic RA examined at our institution using the Myositis Profile 3 kit (Euroimmun AG, Lübeck, Germany). Arguments can be made both for and against this kit in terms of its sensitivity and specificity, as while it can conveniently measure many kinds of anti-ARS antibodies, it cannot measure them all. If a sample tested positive for anti-ARS, we checked for cytoplasmic staining in human epithelial type 2 cells using indirect immunofluorescence. However, the truth is that this confirmation method is not definitive, as there may have been antibodies other than anti-ARS that reacted in the cytoplasm.

Some patients also exhibited Raynaud's syndrome, as you mention; however, the osseous changes observed were characteristic of RA, and not of arthritic symptoms associated with anti-synthetase syndrome. When writing this paper, we believed the diagnosis of RA to be unproblematic, noting that sometimes even patients with RA can test positive for anti-ARS, in which cases a correlation with ILD is observable. Nevertheless, we also agree with the points you have made, and have re-considered our diagnosis of RA in each case. However, one of our anti-PL-12-positive cases has recently shown symptoms of myositis, indicating he might instead have an overlapping syndrome between RA and polymyositis. The present manuscript does not contain detailed data on RA staging or Raynaud's syndrome co-occurrence for each patient. We are planning to re-examine the cases presented here in greater depth, if possible including re-testing of samples for anti-ARS using immunoprecipitation techniques, and conducting careful long-term observation and follow-up. We hope to prepare another report in the future in the event of significant changes in our results after an in-depth examination of the patients during long-term follow-up. Once again, we would like to thank you very much for your valuable feedback.

Correspondence: Masakazu Matsushita, MD. Department of Internal Medicine and Rheumatology, Juntendo University, School of Medicine, 113-8431 Tokyo, Japan.

Tel: 81-3-5802-1067 e-mail: collagen@juntendo.ac.jp 